\title{
Cátedra de la paz colombiana: crítica constructiva al anagrama incompleto ${ }^{1}$
}

\section{Colombian chair of peace: constructive criticism to the incomplete anagram}

\author{
Rafael Guillermo Pizarro Yepes ${ }^{2}$ \\ rpizarroy@gmail.com
}

\section{Resumen}

Con beneplácito, e incertidumbre a la vez, se recibió la noticia de la promulgación de la Ley 1732 de septiembre 1 de 2014, por la cual se estableció "La Cátedra de la Paz" en todas las instituciones educativas del país, sin embargo, ad portas del cuarto aniversario, la magnitud de la temática, la falta de conocimiento e improvisación han complicado la orientación de ésta.

En un país instruido en la guerra, es apenas normal que se haya dificultado enseñar la paz, no obstante, ésta no será enigma imposible de resolver. Aunque desde el año 2016 el Instituto Colombiano para la Evaluación de la Educación (ICFES), en la prueba SABER 11, evalúa la cátedra de paz en la prueba de Sociales y Ciudadanas, tal como reglamenta el decreto 1038 de 2015, aún es perentorio dilucidar el sílabo, la metodología, la población objetivo y la capacitación docente en las instituciones.

Palabras clave: Educación; Cátedra de la Paz; Sílabo; Paz; Metodología; Pedagogía para la paz.

\section{Abstract}

With pleasure, and uncertainty at the same time, the news of the promulgation of the Law 1732 of September 1st 2014, was received, by which "the Chair of Peace" was established in all the educational institutions of the country, however, approaching its fourth anniversary, the magnitude of the subjects, the lack of knowledge and improvisation have complicated its orientation.

In a country so instructed in war, it is evidently normal that Peace has been difficult to teach, however, this will not be an enigma impossible to solve. Although since 2016 the Colombian Institute for the Evaluation of Education (ICFES) assesses the chair of peace with SABER 11 test, in Social Studies and Citizenship test, as regulated by decree 1038 of 2015, it is still peremptory to elucidate the syllabus, the methodology, the target population and the teacher's training at institutions.

Keywords: Education; Chair of Peace; Syllabus; Peace; Methodology; Pedagogy for peace.

'Artículo de reflexión.

${ }^{2} \mathrm{Mg}$. en Estudios de Paz y Conflictos. Docente Fundación Universitaria Monserrate - Unimonserrate. ORCID https://orcid.org/0000-0001-9183-7858

${ }^{*}$ Cómo citar este artículo: Pizarro, R. (2018). Cátedra de la paz colombiana: crítica constructiva al anagrama incompleto. Hojas y Hablas, (15), 34-48. DOI: 10.29151/hojasyhablasn.15a2 


\section{Introducción}

Justo en tiempos de las elecciones presidenciales, de la implementación de acuerdos de paz con grupos insurgentes, del desmantelamiento de redes de corrupción estatales, sobornos y desastres ambientales, de la indebidamente impulsada cátedra de la paz, se vislumbra como una solución a éstos y más flagelos, no sólo para el futuro, representado en la niñez y la adolescencia, sino también para el presente.

Actualmente la cátedra de la paz en las instituciones genera perplejidad, cual logogrifo incompleto o inmarcesible. Aquel pasatiempo, en forma de enigma, que consiste en adivinar varias palabras, con las mismas letras que componen el primer vocablo, a partir de pistas sobre el significado de éstas.

Como un juego que cada quien acomoda a su antojo, de acuerdo a su experiencia, léxico e intelecto, utilizando las mismas letras, así se está planteando la cátedra de la paz. Cada institución la formaliza siguiendo sus principios, percepciones y saberes, sin violar la ley, claro está; ante este evento surgen preguntas inminentes como: ¿cuántas cátedras pueden existir a nivel nacional? ¿Existe control alguno o regulación por parte del estado? ¿Desde el decreto fuente, se generan dudas o vacíos para interpretaciones?

En este artículo se analizará el decreto 1038 de mayo 25 de 2015, por el cual se reglamenta la cátedra de la paz, establecida en la ley 1732 de septiembre 1 de 2014, que, a pesar de incursionar en los viñedos de la educación para la paz como prueba piloto, y es lacónico desde su génesis, no está exento de la crítica constructiva.

Terreno fértil para este proyecto fue la ley 1620 de marzo 15 de 2013, por la cual se crea el "Sistema nacional de convivencia escolar y formación para el ejercicio de los derechos humanos, la educación para la sexualidad y la prevención y mitigación de la violencia escolar", necesario, toda vez que el antiquísimo matoneo, bullying, estaba cobrando vidas y los embarazos a temprana edad se intensificaron.

Comprensiblemente, tantos lustros de maldad, secuela de la dinámica del conflicto interno colombiano, en conjunto con otras problemáticas sociales, agudizaron la violencia tanto en las escuelas como en los barrios.

Estos factores hicieron que el decreto tema de estudio se convirtiera en un bastión curricular por la diversidad de temas neurálgicos y pertinentes que contiene, tales como: 1. Las competencias ciudadanas, ordenadas en tres grandes grupos o estándares (Mineducación, 2004, p. 12), Convivencia y paz, Participación y responsabilidad democrática, y Pluralidad, identidad y valoración de las diferencias; 2 . La memoria histórica, 3. La justicia, 4. Los derechos humanos, entre otros. Sin embargo, la explicación es nula, no existe una guía o manual para llevar la cátedra de la paz a cabo, llega hasta ahí, como una ferrovía inconclusa.

La cátedra de la paz es una idea novedosa, pasaron varios años para que se materializara y ahora que está en sus pinitos, la academia debe aportar el máximo posible de sapiencia para su perfeccionamiento. Solo faltan algunos ajustes, a continuación, unas sugerencias. 


\section{El vago decreto}

La Cátedra de la Paz, reglamentada por el decreto 1038 de mayo 25 de 2015, debe adscribirse dentro de alguna de las siguientes áreas fundamentales, establecidas en el artículo 23 de la ley 115 de 1994: “a) Ciencias Sociales, Historia, Geografía, Constitución Política y Democracia; b) Ciencias Naturales y Educación Ambiental, o c) Educación Ética y en Valores Humanos".

Limitar la paz en estas asignaturas es un yerro, ésta complementa cada una de las nueve áreas obligatorias y fundamentales (Ley 115 de Educación, 1994, art. 23). Inclusive los docentes de matemáticas podrían hacer un aporte valioso a la paz, así como lo ha hecho Johan Galtung, de quien hablaré posteriormente. Más si su temática es tan extensa, ¿será que la paz no amerita ser estudiada como una disciplina o materia independiente?

Desde la Constitución Política de Colombia de 1991 se plantean rudimentos para los estudios de paz, los artículos 22, 67 y 95 hablan de paz, como deber y derecho, y de la educación como derecho y su énfasis en el respeto los derechos humanos, a la paz y a la democracia; y en la práctica del trabajo y la recreación, para el mejoramiento cultural, científico, tecnológico y para la protección del ambiente.

Uno de los fines de la educación, contemplado en el artículo 5 numeral (2) de la Ley 115 de 1994 (Ley General de Educación), fomenta "la formación en el respeto a la vida y a los demás derechos humanos, a la paz, a los principios democráticos, de convivencia, pluralismo, justicia, solidaridad y equidad, así como en el ejercicio de la tolerancia y de la libertad", asimismo, el artículo 14 literal (d), establece la obligatoriedad de enseñar en todas las instituciones públicas y privadas "la educación para la justicia, la paz, la democracia, la solidaridad, la confraternidad, el cooperativismo y, en general, la formación en los valores humanos..."

Las bases sólidas que hay en las leyes colombianas permiten moldear proyectos educativos transversales incluyentes, donde la esencia son los derechos humanos y la convivencia, como los de educación campesina y grupos étnicos (etnoeducación) y educación para personas con limitaciones o capacidades excepcionales. No obstante, sólo hasta el año 2015 se materializó la cátedra de la paz, con miras al perfeccionamiento.

Pese a que el decreto de dicha cátedra es impreciso y da cierta libertad a las instituciones educativas de adecuar la cátedra a sus creencias, principios o proyecto educativo institucional (PEI), y se podría tergiversar el tema central, es un gran acierto delimitar la cátedra a doce temas.

De acuerdo con Coll (1991, p. 173) un currículo debe ofrecer información bien respaldada, que permita responder las preguntas: ¿qué?, ¿cuándo?, ¿cómo enseñar y evaluar? Sin duda la cátedra debe fundamentarse en mínimo tres pilares: metodología, sílabo o temática, y población, cimientos carentes en éste mandato en unos casos e incompletos en otros.

Las ambigüedades abren la portilla al riesgo de ocasionar dificultades y frustraciones en la formación, tanto a docentes como a estudiantes que, al fin y al cabo, repercutirán en la sociedad; haciendo una reflexión sobre la temática de un sólo literal del decreto, por ejemplo, el d) Resolución pacífica de conflictos, ¿¿cuántos subtemas se podrían tratar?

Antes de exponer las tres dimensiones o 
pilares, es necesario aclarar los términos enseñar y aprender. De lo escuchado en una conferencia sobre la evolución de la educación, liderada por el expositor español Ismael Peña-López (2016), experto en Conflictología, puedo parafrasear lo siguiente:

Anteriormente el acceso a la información era precario y por ende el conocimiento. Eran muy pocos los que tenían contacto con los expertos o con los libros. Actualmente, gracias a invención de la tinta y de la imprenta, muchos eruditos pudieron reproducir el saber, es decir, transmitir conocimiento (enseñar). La expedición de libros facilitó el proceso de aprendizaje, pero el internet definitivamente fue el boom. Con la información tan cerca, el proceso de adquirir conocimiento (aprender), se simplificó”.

Los modelos pedagógicos tradicionales, algunos basados en la memorización, otros con un tinte violento, han evolucionado, ahora se habla de aprender y no tanto de enseñar. Por lo visto en los salones de clase, parece que la enseñanza obsolescente sucumbirá ante el imponente e inevitable perfeccionamiento del aprendizaje, que es, la producción de conocimiento. Pedro Gutiérrez Leal manifiesta (2004):

Para interpretar el origen del conocimiento, partiremos por analizar la relación fundamental que se establece entre el hombre y el mundo real. $\mathrm{Su}$ necesidad básica es sobrevivir, al igual que la de cualquier otro organismo. Necesidad ésta que es posible comprender objetivamente a través de la cantidad requerida y del tiempo que le toma conseguirlo. Cuando el desarrollo del conocimiento y las técnicas para producir son escasas, la cantidad es limitada y el tiempo es mayor. De esta manera el ser humano debe invertir mucho esfuerzo y someterse a grandes riesgos.
Nuestra curiosidad y necesidad nos llevarán a pensar, inventar y a producir saber, así como cuando hubo la necesidad de sembrar, cazar o pescar. En el caso del fuego, indistintamente de su invención, accidentada o deliberada, después de descubierto, se supo de sus utilidades y que este no se podía agarrar con las manos o siquiera tocar porque quemaba, ahí se produjo conocimiento. Con la aparición del internet y las redes sociales, la transferencia de información y saberes se hizo espontánea, antes se necesitaba de un mediador entre los recursos (conocimiento) y el discente (estudiante), así como un espacio y tiempo determinados, que ya no son tan requeridos. Ahora el profesor, maestro o docente, cumple otras funciones, no solamente la de "depositar" conocimientos como lo manifestó Freire (1974, p. 58).

Una vez clara la diferencia entre enseñanza (transmitir saberes) y aprendizaje (adquirir conocimientos), el decreto debe exponer, como mínimo, qué aprender, y especialmente, cómo.

\section{La temática, la población y la capacitación docente}

¿Qué aprender, para qué, con quién y para quién? Esa es la cuestión. Según el matemático y sociólogo Galtung (1969, p. 167), para muchos el padre de los estudios de paz y conflictos, una máxima de la paz es la ausencia de violencia, pero sin duda, la paz es mucho más que eso. Él define tres tipos de violencia (Galtung, 2000, p. 7 ), violencia directa, estructural y cultural. La violencia directa se manifiesta al lastimar intencionalmente, física o psicológicamente, o al matar. La violencia estructural es aquella llevada a cabo por las estructuras de la sociedad, gobierno, empresarios, militares, y otras, donde de manera injusta no se evitan los sufrimientos o la muerte 
lenta por hambruna, acoso sexual o laboral, enfermedades curables u otras causas, debido a la negligencia, corrupción, falta de libertad, empatía y democracia. La violencia cultural es aquella manifestada a través de nacionalismo, racismo, sexismo, bullying o matoneo, prejuicios en educación, medios de comunicación, literatura, cine, arte, música y otros factores culturales.

Hay muchas definiciones y percepciones relacionadas con la paz, desde varios ángulos, latitudes y autores, por ejemplo, la paz interior o interpersonal, donde juegan un papel importante el yoga, la meditación, la religión, la libertad de expresión, de movimiento financiero o de las deudas; está la paz de los victoriosos o vencedores, que se refiere al botín de guerra y otros beneficios; la paz mundial y el concepto de paz de los ambientalistas, líderes religiosos, pueblos indígenas, dictadores, países desarrollados, en vía de desarrollo, subdesarrollados, entre otros. Éstas definiciones, de una u otra forma, me causan confusión no obstante, existen aspectos claves en cada opinión que necesitan ser mencionadas, con el fin de completar un concepto o definición. Seguramente el concepto de paz de un habitante de calle es diverso al de un maestro de yoga, pero ambos tendrán puntos ciertos y vinculantes al término.

Para hablar de paz y como construirla, Peacebuilding, en Colombia o en cualquier otra nación, es necesario tener claro que la paz puede ser vista desde dos frentes, como un estado, de concordia, felicidad, tranquilidad y salud de la mente y el cuerpo, y como un "paso a paso", que abarca la capacidad de transformar conflictos con empatía, creatividad y sin violencia, un proceso de nunca acabar (Galtung, 2010, p. 91). Con respecto a éste último, se podría sintetizar que un proceso de paz es:
1. Recordar los hechos importantes que han ocurrido e investigar a profundidad la causa raíz del conflicto, estudiando la historia y analizando las diferentes contradicciones y partes del conflicto, tanto las más visibles como las invisibles, afectadas indirectamente, en los distintos niveles de la sociedad; evaluando las iniciativas en pro de la paz, es decir, validar lo que se ha hecho hasta el momento, enmendar los errores cometidos y generar nuevas propuestas.

Aunque el análisis y la evaluación toman tiempo, son la base fundamental para empezar la reconstrucción de una nueva realidad, una sólida, duradera y estable. El análisis y la evaluación son pasos esenciales para la generación de buenas soluciones.

2.Después de encontrar la base del conflicto, la paz puede ser construida si incluye, la ausencia de todos los tipos de violencia, la satisfacción de las necesidades básicas (alimentación, educación, salud, oxígeno puro, agua potable, vivienda, libertad de expresión, entre otras.), respeto a los derechos humanos y la justa distribución de poder, recursos y oportunidades, entre otros conceptos.

Sin embargo, el proceso de la paz no solo consiste en practicar las premisas antes mencionadas, sino también la búsqueda de una solución o transformación al conflicto, donde las partes incluyendo la naturaleza - puedan coexistir sin abusos entre sí.

3.Finalmente, se debe incluir en este proceso "sanar el pasado", el cual permitirá sostenibilidad, a través del perdón, unidad, inclusión, reintegración y reconciliación. 
Después de ésta sucinta introducción a la paz y retornando al eje fundamental de análisis, se evidencia que todos los temas de la cátedra son vitales para la consecución de una cultura de paz, aunque escueta, porque necesita ampliar su arista curricular, ciertamente el dubio se genera, por un lado, cuando se manifiesta que se deben desarrollar al menos dos de los doce capítulos, quitando así plenitud a la asignatura, y por otro, cuando se desconoce la extensión o subtemas de la materia; irremisiblemente esto incita a la práctica natural y pedagógica del ensayo y error. Esto dice la ley en cuanto al currículo:

Artículo $4^{\circ}$. Estructura y contenido. Los establecimientos educativos de preescolar, básica y media determinarán los contenidos de la Cátedra de la Paz, los cuales deberán estar orientados al logro de los objetivos consagrados en el parágrafo $2^{\circ}$ del artículo $1^{\circ}$ de la Ley 1732 de 2014 y en el artículo $2^{\circ}$ del presente decreto y deberán desarrollar al menos dos (2) de las siguientes temáticas:
a) Justicia y Derechos Humanos;
b) Uso sostenible de los recursos naturales;
c) Protección de las riquezas culturales y natu- rales de la Nación;
d) Resolución pacífica de conflictos;
e) Prevención del acoso escolar;
f) Diversidad y pluralidad;
g) Participación política;
h) Memoria histórica;
i) Dilemas morales;
j) Proyectos de impacto social;
k) Historia de los acuerdos de paz, nacionales e internacionales;
1) Proyectos de vida y prevención de riesgos (Decreto 1038 de 2015, artículo 4)
No fue un exabrupto confinar la cátedra de la paz a doce temáticas, ya que la paz es un tema extenso e importante en su plenitud, por tanto, las unidades dadas deben detallarse un poco más; y con mayor ahínco si se elige orientar solo dos temas.

En la Escuela de Sub-oficiales de la Fuerza Aérea Colombiana (ESUFA), dando cumpliendo a la orden, se orientan dos temas de la cátedra, Historia de los acuerdos de paz, nacionales e internacionales, con los subtemas: Proceso de paz en Colombia, acuerdo inicial, desarrollo de la agenda, rol y retos de las FF.MM. y de la sociedad civil en los diálogos de paz y eventual posconflicto y memoria histórica, la cual abarcaría la contextualización histórica, causa y origen del conflicto armado. A mi parecer, las competencias en resolución de conflictos, justicia, derechos humanos y en general todas, son relevantes, tanto para la milicia como para los demás; saber de memoria histórica o protección de riquezas culturales, se complementaría con la capacidad de resolver conflictos pacíficamente, y para hacerlo, se requieren conocimientos mínimos en derechos humanos, comunicación asertiva, dilemas morales, diversidad, pluralidad, uso sostenible de recursos naturales, justicia y otros aspectos. Sería triste saber de proyectos de vida, de impacto social y prevención de riesgos y no tener capacidad para resolver un conflicto en casa.

Por eso, cátedras similares, nacionales e internacionales, de las cuales hablaré posteriormente, recalcan la importancia del aprendizaje integral, detallando los sílabos al máximo.

Entretanto, Natalia Durán, en su artículo "Educar para la paz, no para una cátedra", relata algunas de las intervenciones del Encuentro Colombia 2020 sobre "La educación como pilar 
fundamental para la construcción de paz", de las cuales resalto la reflexión de Annika Otterstedt, jefa de cooperación de la Embajada de Suecia en Colombia, quien dijo: “ $¡$ Por qué en los colegios no se habla del Acuerdo de Paz al que llegó el Gobierno con las Farc para poner fin a 52 años de conflicto armado que dejó millones de desplazados y miles de colombianos muertos? Otterstedt se preguntó con sorpresa por qué a sus hijos que estudian en el país no les hablan de este tema que no pocos califican de histórico y trascendental". Estas palabras son viva muestra que la cátedra, desde la perspectiva de una madre, está incompleta, por lo menos en la(s) institución(es) donde estudian sus hijos.

Es recomendable y pertinente especificar los subtemas que son menester, por lo menos para primaria y bachillerato, máxime si los rectores, o quienes cumplan funciones de expertos curriculares, no son doctos en estudios de paz y conflictos. En el artículo 7 del decreto 45 de 1962, se especifica la asignación de ciertos temas de acuerdo a la asignatura y al grado, lo mismo debería pasar con la cátedra de la paz, por lo menos detallar los subtemas a impartir en la educación básica primaria, secundaria y media vocacional, para así esclarecer el modus operandi, pues como están los temas tipificados actualmente, resultan abiertos y da cabida a un sinnúmero de interpretaciones. Se sugiere una organización de temas por competencias.

A manera de ejemplo, un tipo de organización de posibles subtemas, basados en competencias cognitivas (gestión del conocimiento y solución de problemas), emocionales, comunicativas, sociales (trabajo en equipo y compromiso social) e integradoras, siendo la cátedra de la paz una asignatura emancipada, para séptimo grado, podría ser la siguiente:
Parte I: Competencias esenciales para la paz. Incluye conceptos, teorías y herramientas para la paz, tiene el firme propósito de revelar mayor y mejor conocimiento, tácticas y técnicas para la solución pacífica de conflictos, lo cual permitirá empezar a llenar la caja de herramientas de la paz con implementos, en otras palabras, con experiencias y sapiencias. Algunos de los subtemas podrían ser:

Capítulo 1: Conflicto, 1.1 Tipos y causas básicas del conflicto, 1.2 Actitudes y respuestas al conflicto, 1.3 Mecanismos de manejo de conflictos, 1.3.1 Negociación, Mediación y Métodos Alternativos de Solución de Conflictos (MASC), 1.3.2 Transformación y transcendencia, 1.3.3 Herramientas TRANSCEND para el análisis en la transformación de conflictos, 1.3.4 Otras herramientas, estrategias y consejos para solucionar conflictos pacíficamente, Capítulo 2: Necesidades Básicas, 2.1 Derechos Humanos, 2.1.1 Derechos de los niños, Capítulo 3: Violencia y No-violencia, 3.1 Tres tipos de violencia, 3.2 No-violencia, Capítulo 4: Paz, 4.1 Perfil o características de un gestor de Paz.

Parte II: Competencias especiales para la paz. Se enfoca en la evasión del círculo vicioso que traen la violencia y la venganza, que, a su vez, impiden el crecimiento y el progreso de una persona, familia, comunidad o nación. Esta parte tiene como propósito encontrar soluciones buenas, reales y sostenibles, para de una vez por todas se pueda evolucionar del manejo del conflicto a la prevención de la violencia. Entre los temas a estudiar en esta competencia están:

Capítulo 5: Perdón, Capítulo 6: Reconciliación, unidad e inclusión, 6.1 Unidad, 6.1.1 Diversidad y pluralidad, 6.2 Inclusión, 6.2.1 Bullying y prevención del acoso, 6.2.2 Reintegración, 6.3 
Etnoeducación y formación incluyente, 6.4 Justicia y participación, 6.4.1 Justicia Transicional, 6.4.2 Preservación de la memoria histórica, 6.4.3 Participación política y democrática.

\section{Parte III: Competencias personales para la paz. Esta parte se organiza así:}

Capítulo 7: Creatividad para la paz, $7.1 \mathrm{El}$ proceso de la creación, 7.2 Distorsiones de la creatividad, 7.3 Pensamiento lateral y vertical, 7.4 Herramientas para el pensamiento creativo, Capítulo 8: Comunicación para la paz, 8.1 Escucha activa, 8.2 Retroalimentación positiva y crítica constructiva, 8.3 Asertividad, 8.4 Modelos y estilos de comunicación, 8.5 Lineamientos para una comunicación efectiva, Capítulo 9: Valores, principios y sentimientos.

La carencia o debilidad en algunos de los conceptos es fuente de violencia o conflicto. El capítulo Creatividad para la Paz, busca desarrollar la importante capacidad humana de la creación, especialmente la creación de métodos para desenredar contradicciones, promover la búsqueda de nuevas maneras de pensar y ver las cosas, producir más y mejores ideas, abrir la mente para la construcción de una nueva y mejor vida, libre de violencia, que a la postre, es el resultante de la falta de creatividad, comunicación o valores. Este fragmento tiene por objeto mejorar la capacidad de idear, pensar y construir métodos alternativos de solución pacífica de conflictos, primordialmente en los niños y niñas de Colombia.

El capítulo Comunicación para la Paz, promueve el análisis y la identificación de las necesidades básicas y legítimas por medio de distintos modelos y estilos de comunicación, así como fortalecer la habilidad de la comunicación asertiva y escucha activa, especialmente en aquellos que carecen de habilidades en comunicación verbal y no verbal.

Los valores, principios y sentimientos, incentivan la construcción de una sociedad con valores, compuesta de diversas comunidades, cada una con sus creencias, donde las opiniones son siempre respetadas y en ningún caso criticadas, ridiculizadas, estigmatizadas o presionadas a cambiar, a pesar de no ser aceptadas, comprendidas o compartidas. Buscan por supuesto fortalecer esta habilidad en todos, niños y adultos, que carecen de empatía, tolerancia, solidaridad, compasión y simpatía.

\section{Parte IV: Liderazgo y gestión de equipos para} la paz. La cuarta parte, indaga sobre los temas y estrategias para un liderazgo inclusivo, estudia conceptos importantes como:

Capítulo 10: Liderazgo, 10.1 Poder, 10.2 Autoridad, 10.3 Legitimidad, 10.4 Distorsiones del liderazgo, 10.4.1 Corrupción, Capítulo 11: Gestión de equipos y liderazgo inclusivo, 11.1 Estilos de liderazgo y evolución hacia la paz, 11.2 Liderazgo transformacional, 11.3 Liderazgo interpersonal para la paz, 11.3.1 Gana - Gana, 11.3.2 Motivación y empoderamiento

Capítulos que buscan cultivar líderes para la paz, quienes tienen un idóneo entendimiento de los siguientes tres elementos: poder, autoridad y legitimidad; frecuentemente malinterpretados y abusados.

De igual forma, enseña a distinguir entre tres estilos de liderazgo: rígido, relajado e inclusivo; motivando siempre a desarrollar y aplicar el último.

La sección Gestión de equipos y liderazgo 
inclusivo, fomenta los principios de unidad y cooperación para satisfacer necesidades e intereses comunes que apuntan al desarrollo humano, cambiando de un enfoque gana-pierde a gana-gana.

Parte V: Preservación del medio ambiente y desarrollo sostenible. La última parte muestra algunas de las claves para convivir en sociedad, incluyendo la naturaleza. Algunos de los subtemas podrían ser:

Capítulo 12: Desarrollo, convivencia y medio ambiente, 12.1 Convivencia social, Capítulo 13: Desarrollo sostenible, 13.1 Uso sostenible de los recursos naturales, 13.2 Objetivos del Milenio ONU, 13.3 Consejos para preservar el medio ambiente, Capítulo 14: Protección de las riquezas culturales y naturales de la nación.

En mi opinión y teniendo en cuenta las experiencias exitosas de otras cátedras y programas de estudios de paz, los doce temas que sugiere el decreto son suficientes para empezar, y se podrían desagrupar en subtítulos, subtemas o capítulos, vincular a las partes del ejemplo previo u organizar de esta manera, por competencias, para mayor claridad. Así como lo hizo la World Peace Academy, hoy SwissPeace, y la Universidad Europea para la Paz (EPU), que detallaron los subtemas de los sus Maestrías en Paz y Transformación/Resolución de Conflictos, basándose en los múltiples textos de Johan Galtung y otros autores; actualmente el Instituto Galtung y la Universidad para la Paz Transcend, se anclan en la obra del fundador y especialmente en los tres pilares o tipos de violencia y paz, directa, estructural y cultural, para establecer su temática.

La cátedra del Instituto Internacional de
Educación para la Paz, usa los capítulos del libro de H.B. Danesh, Educación para la Paz: Manual Curricular, y la Universität Jaume I (UJI), cimenta los módulos de su Maestría en Paz en el marco la cátedra UNESCO de filosofía para la paz.

Todos estos programas internacionales, entre otros, especifican los subcontenidos y se apoyan en expertos, para escoger temas claves, afines a los ya mencionados. En Colombia, la cátedra de paz del Servicio Nacional de Aprendizaje SENA, se orienta a través de tres programas de formación complementaria, Ciudadano promotor de paz, Formadores en cultura de paz y Gestor de paz, que mencionan los subcapítulos.

A las instituciones de educación superior, por el principio de autonomía universitaria, se les permite desarrollar la Cátedra de la Paz en concordancia con sus programas académicos y su modelo educativo. Tal y como reza el artículo noveno del decreto 1038 de 2015:

Artículo $9^{\circ}$. Instituciones de Educación Superior. En desarrollo del principio de la autonomía universitaria, las instituciones de educación superior desarrollarán la Cátedra de la Paz en concordancia con sus programas académicos y su modelo educativo, para lo cual podrán definir las acciones educativas que permitan a la comunidad académica contar con espacios de aprendizaje, reflexión y diálogo para la vivencia de la paz (Decreto 1038 de 2015, artículo 9).

Las universidades, con su irrebatible libre albedrío, no están sufriendo con este tema, los colegios en cambio, podrían estar padeciendo e improvisando.

Entender las comunidades de aprendizaje 
es una tarea fundamental. Se cree que la cátedra es sólo para estudiantes de educación básica primaria, secundaria, media y superior, tal como se instituyó, inusitadamente se habla de los prospectos en los tres poderes del estado, en las empresas multinacionales, en las asociaciones de padres de familia, juntas de acción comunal, sindicatos, etc., todos necesitamos capacitación sobre paz; y de la población emana la metodología, próximo subtítulo.

Por otra parte, los docentes, también deben ser entrenados para ser formadores de paz en su asignatura. Indistintamente de sus áreas de experticia o metodología en clase, éstos pueden desempeñarse como multiplicadores, así lo sostiene el artículo séptimo:

Artículo $7^{\circ}$. Capacitación y Formación Docente para la Cátedra de la Paz. Las entidades territoriales certificadas en educación, en trabajo articulado con los Comités Territoriales de Capacitación a Docentes y Directivos Docentes, deberán:

a) Identificar cada dos (2) años las necesidades de formación de los docentes y directivos docentes en servicio adscritos a la entidad territorial en materia de Derechos Humanos, cultura de paz, y competencias ciudadanas para la convivencia pacífica, la participación democrática, la diversidad y pluralidad;

b) Financiar o diseñar en sus respectivos planes de formación a docentes y directivos docentes, programas y proyectos de alta calidad que ofrezcan las instituciones de educación superior y otros organismos, para responder a los objetivos de la Cátedra de la Paz, así como promover su incorporación a los mismos;

c) Valorar y evaluar cada dos (2) años, mediante mecanismos adecuados y contextualizados, el impacto de los programas y proyectos de formación a docentes y directivos docentes.

Parágrafo. El Ministerio de Educación Nacional promoverá el desarrollo de estrategias para la formación específica de los docentes y directivos docentes, orientados a educar en una cultura de paz y el desarrollo sostenible, conforme con los lineamientos de la Cátedra de la Paz. (Decreto 1038 de 2015, artículo 7)

Un currículo efectivo y una metodología idónea, basada en la población, son condiciones sine qua non para adquirir y/o fortalecer las competencias ciudadanas (Mineducación, 2004, p. 8), que "son el conjunto de conocimientos y de habilidades cognitivas, emocionales y comunicativas que, articulados entre sí, hacen posible que el ciudadano actúe de manera constructiva en la sociedad democrática”. Una temática robusta y metodología seductora, encauzadas por un enfoque pedagógico ideal para la paz, que podría ser axiológico-altruista u otro, son dos de las columnas faltantes en la ley.

\section{La metodología sugerida}

La metodología responde la pregunta cómo aprender. La pedagogía para la paz, debe ser una propuesta ambiciosa. Un buen método, asiduo en uso por sus altos niveles de aceptación y aprendizaje, será aquel que ponga en práctica "aprender haciendo", y mejor aún, "aprender jugando y riendo"; independiente de la edad.

Según la dimensión cognitiva del modelo pedagógico humanista-cognitivo del Servicio Nacional de Aprendizaje SENA, el aprendizaje "se desarrolla de manera autónoma por parte del aprendiz, con el propósito de ser aplicado 
a la solución de problemas, evidenciando de esta forma el desarrollo de competencias" (PEI SENA, 2013, p. 5), es decir, por más que se trate de inyectar sabiduría o conocimiento, la persona misma, con sus estilos de aprendizaje, lo adquiere. Asimismo, la orientación humanista, social y educativa de la Fundación Universitaria Monserrate - Unimonserrate, evoca el foco de toda pedagogía, el ser humano y sus múltiples dimensiones (PEI Unimonserrate, 2016, p. 18).

Por otra parte, La Corporación Universitaria Minuto de Dios ha adoptado un enfoque pedagógico praxeológico que integra el saber (teoría) y la práctica (praxis) mediante un proceso reflexivo que parte del análisis crítico de las prácticas y experiencias de cada persona o comunidad, llevándolas a integrar su(s) proyecto(s) de vida personal y profesional, a un proyecto de transformación de la sociedad". (Uniminuto, 2014, p. 65)

Los tres modelos educativos se apoyan en enfoques enmarcados en el desarrollo humano, con el fin adquirir y/o fortalecer las competencias básicas (ser, hacer y saber), y las específicas o profesionales, para actuar con responsabilidad social. A pesar de que existen múltiples modelos pedagógicos como el conductista, romántico, social, entre otros, para la cátedra de paz, el estandarte debería ser un enfoque axiológico, cimentado en valores y principios, legítimos y universales, con un fin altruista o similar, es decir, al servicio de los demás, o de todos (incluyéndome), pues de nada servirá todo el conocimiento adquirido si es para causar daño a la sociedad, al planeta o para satisfacer necesidades y deseos netamente propios.

Se deduce del decreto, que respeta la libertad de cátedra en cuanto a la metodología, así como se consienten los modelos pedagógicos de las instituciones, ya que no se refiere a ello. Empero, la propuesta de paz debería seguir ciertos parámetros, como desarrollarse en un ambiente libre de presiones, centrado en el desarrollo de competencias ciudadanas para la paz, que presente un carácter problémico, sistémico, integrador e interdisciplinario, evidenciado en el bien a los demás, que contenga actividades como: Teatro, competencia sana, actividades con pintura, ejercicios de auto-superación, creación de canciones, actividades de motivación a explorar y cuidar la naturaleza, estrategias gana-gana, estudio de testimonios, vivencias, debates, experiencias, historias, relatos, entre otras técnicas dinámicas. Todas estas actividades, grupales o individuales, deben amoldarse y explicarse desde el contexto de la cátedra de la paz. Por ejemplo, si se está haciendo una carrera en sacos, esta debe tener relación con al menos uno de los capítulos o partes de la temática, y después de la recreación, se dará una pequeña justificación teórica, y lo mejor de todo, en la medida de lo posible, fuera de las cuatro paredes estigmatizadoras de un salón de clases.

Las didácticas para la paz, aterrizadas en el contexto latinoamericano, toman valía al momento de la enseñanza, Jeffrey Sobel, La Fundación Nuevos Juegos (The New Games), entre otros, han desarrollado juegos no competitivos como una alternativa, convencidos que "todos podemos ganar”. Asimismo, los programas lúdicos de paz de Peace Games y Peace by PEACE, originados en 1980 por la Doctora Francelia Butler de la Universidad de Connecticut, promueven la solución de conflictos en niños, de manera divertida, donde no hay ganadores ni perdedores, solo recreación con aprendizaje.

Otras dinámicas como el teatro del oprimido, de 
Augusto Boal (basado en Freire) y muchas otras estrategias metodológicas, se amoldan a la cátedra de paz.

Esta metodología, sugerencia para las instituciones y no para el decreto, resultado de varias experiencias exitosas y programas en los que he participado, utilizada en cualquier momento de la formación, como reflexión inicial, apropiación o transferencia de conocimiento, evaluación o conclusión, se puede complementar con otras estrategias metodológicas como el aprendizaje por proyectos, y otras didácticas activas, asimismo, con varios estilos de aprendizaje tales como el quinestésico, visual, autónomo, colaborativo, significativo, o los más representativos de Kolb (1974, p. 38), divergente, asimilador; convergente y acomodador o adaptador; en la paz no hay camisas de fuerza.

Los estudiantes de la cátedra de la paz también pueden adquirir conocimientos por sus medios, con sus vivencias, agradables o traumáticas. Esta asignatura es difícil aprenderla de memoria, sin práctica o enseñada. La asistencia y motivación del docente cobran gran importancia, y aunque el docente cumpla funciones de tutor, mediador y evaluador, todos tienen algo que ofrecer a la hora de aprender, de hecho, de todos se aprende.

El método motiva la búsqueda y aplicación de esa gran variedad de ejercicios dinámicos que ya existen, o a la creación de nuevos, que faciliten el desarrollo de las diferentes áreas del crecimiento personal: Física, intelectual, afectiva, social y espiritual, y como resultado del desarrollo de estas áreas, el carácter o identidad (The World Scout Bureau, 2005, p. 23).

Este enfoque, dinámico, divertido, atractivo e innovador, permitirá la aceptación de la cátedra de la paz con facilidad y colaborará sin duda alguna, al florecimiento de la educación para la paz en Colombia y motivará a los participantes a debatir y a contribuir a la creación de un mejor país. Además, la ley 1098 de 2006 (Ley de Infancia y Adolescencia), en su artículo 30, derecho a la recreación y participación en la vida cultural y artística, lo auspicia.

Y con respecto al control, cuestionado en la introducción de este artículo, sí existe, por lo menos en teoría:

Artículo $8^{\circ}$. Lineamientos y articulación con el Sistema Nacional de Convivencia Escolar. Los Comités de Convivencia Escolar, definidos en la Ley 1620 de 2013, en sus niveles nacional, territorial y Escolar, realizarán seguimiento a lo dispuesto en el presente decreto; a fin de asegurar que la Cátedra de la Paz cumpla los objetivos consagrados en el artículo $2^{\circ}$ del presente decreto (Decreto 1038 de 2015, artículo 8).

Tanto la metodología como la temática deben estar sujetas a constante y exhaustiva evaluación y monitoreo, porque como dice William Thomson Kelvin "Lo que no se define, no se puede medir. Lo que no se mide, no se puede mejorar. Lo que no se mejora, se degrada siempre”, así como se hace con la cátedra:

Artículo $5^{\circ}$. Evaluación. A partir del año 2016, el Instituto Colombiano para la Evaluación de la Educación (ICFES) incorporará dentro de las Pruebas Saber 11, en su componente de Competencias Ciudadanas, la evaluación de los logros correspondientes a la Cátedra de la Paz.

Adicionalmente, el ICFES deberá incorporar gradualmente el componente de 
Competencias Ciudadanas dentro de alguna de las pruebas de evaluación de calidad de la educación básica primaria y de la básica secundaria, según un criterio técnico (Decreto 1038 de 2015, artículo 5).

Si bien las pruebas ICFES-SABER 11 evalúan las competencias cognitivas ¿quién evalúa la práctica? Ahí es donde la sociedad entera entra en acción, como formadora o gestora de paz, que evita la indiferencia y educa cuando se esté arrojando basura en las calles, cruzando un semáforo en rojo, actuando con violencia o en situaciones similares dentro del hogar o en la calle. Sin embargo, aunque este artículo hable de la evaluación que hace el estado, los colegios no son ajenos a este tema, éstos deben establecer evaluaciones cualitativas y cuantitativas, en las relaciones fuera y dentro del salón de clase, con ejercicios individuales y grupales, donde se puedan ver y corregir las reacciones bajo presión.

Ante el escepticismo y pesimismo circundantes, y generalizados, se levanta una tropa optimista pregonando que la esperanza es lo último que se pierde, como manifiesta el viejo adagio. Y sí, la paz es posible, difícil, pero posible. El logogrifo lo podemos completar entre todos, la educación nos compete a todos, y de igual forma la paz.

\section{Aires de paz y esperanza}

No cabe duda que esta ley es el resultado del auge de los estudios de Paz a nivel mundial, es bien sabido que Colombia no es el único país afectado con la fusta de la guerra y demás matices de la violencia. Las consideraciones finales de este artículo, después del análisis, se enmarcan en la necesidad de supervisión, capacitación, apoyo y ajustes que podría hacer el Estado, detallando más la cátedra, en forma y fondo, y en las iniciativas o compromisos de las escuelas, adoptando la metodología y sílabo sugeridos o similares, teniendo presente que la formación está dirigida a estudiantes y profesores.

En espera del eventual y tan anhelado fin del conflicto armado con todas las guerrillas colombianas, la sociedad se está preparando para empezar a construir paz, y las instituciones educativas juegan rol primordial, porque el conflicto no termina en la firma de acuerdos, es el punto de partida para la construcción de la paz.

Diversas instituciones, y en especial las universidades, se están contagiando de paz. Muestra de esto, es la apertura de la "Maestría en Paz, Desarrollo y Ciudadanía" por parte de la Universidad Minuto de Dios, quien dio la noticia el 14 de marzo de 2016 a través de su boletín informativo; se suma a la iniciativa de la Universidad de Pamplona, quien hace años cuenta con la maestría, y a muchas otras instituciones que cuentan con especializaciones y maestrías relacionadas, con diferentes nombres, dedicadas a la solución pacífica de conflictos, como es el caso de la Universidad Externado de Colombia y su "Maestría en Análisis de Problemas Políticos, Económicos e Internacionales Contemporáneos”.

Ahora bien, la esperanza es lo último que se pierde y no todo está perdido, hay ganas y vientos de cambio y de paz, ahora ¿qué pasaría si se tienen más y mejores herramientas, conocimientos o experiencias para resolver conflictos pacíficamente? ¿Qué tal si toda Colombia es orientada en solucionar sus conflictos sin violencia desde temprana edad?

Para conseguir este objetivo, no sólo las 
entidades escolares deben apropiarse de la tarea, todos somos responsables, todos. Este tema novedoso, retador, interesante y provechoso para la nación está en manos de la familia, de los colegios públicos y privados, de las universidades, inclusive de las empresas y entidades del estado.

La cátedra de la paz es un avance valioso, esta propuesta seguramente ayudará a construir y fomentar una cultura de paz en Colombia y colaborará con el florecimiento de los estudios de paz y conflictos, además fortalecerá las competencias necesarias para la resolución pacífica de conflictos a nivel intra- e interpersonal, tales como creatividad, comunicación, empatía, tolerancia, convivencia, liderazgo, análisis de conflictos, prevención contra cualquier tipo de violencia y de corupción, perdón y reconciliación.

Esta cátedra promueve la investigación y activación de nuevas ideas, procesos, herramientas y estrategias para resolver conflictos sin recurrir a la violencia, para éstas y las próximas generaciones; motivará la creación y capacitación de agentes de paz, promotores o facilitadores, quienes examinarán los capítulos aquí divulgados, con el fin de aclarar, actualizar, complementar, transmitir y/o reproducir aspectos claves y conocimientos para una mejor y fácil comprensión, aplicación y explicación de la teoría y las actividades para la paz; solo hay que ajustarla.

\section{Referencias}

Coll, C. (1991). Aprendizaje escolar y construcción del conocimiento. Barcelona: Paidós.

Congreso de la República de Colombia. Ley 1732 de 2014: Por la cual se establece la catedra de la paz en todas las instituciones educativas del país.
Congreso de la República de Colombia. Ley 115 de 1994: Por la cual se expide la ley general de educación.

Congreso de la República de Colombia. Ley 1620 de 2013: Por la cual se crea el sistema nacional de

convivencia escolar y formación para el ejercicio de los derechos humanos, la educación para la sexualidad y la prevención y mitigación de la violencia escolar.

Congreso de la República de Colombia. Ley 1098 de 2006 (Ley de Infancia y Adolescencia).

Constitución Política de Colombia de 1991.

Corporación Universitaria Minuto de Dios Uniminuto. (2014). Proyecto Educativo Institucional del Sistema. Bogotá: Uniminuto.

Durán, N. (2017, mayo 29). Educar para la paz, no para una cátedra. Recuperado de: https:// colombia2020.elespectador.com/pedagogia/educar-para-la-paz-no-para-una-catedra

Freire, P. (1974). Pedagogy of the Oppressed. New York: The Seabury Press.

Fundación Universitaria Monserrate. (2016). Proyecto Educativo Institucional PEI: Proyección -

cercanía - cuidado. Bogotá: Unimonserrate.

Galtung, J. (2000). Conflict Transformation by Peaceful Means: The TRANSCEND Method (Participants' and trainers' Manual). Geneva: United Nations.

Galtung, J. (2010). A theory of conflict: Overcoming direct violence. Oslo: Kolofon press.

Gutiérrez Leal, P. (2004, febrero 10). Produccióndel conocimiento. Recuperado de https:// www.gestiopolis.com/produccion-del-con- 
ocimiento/

Kolb, D. A., \& Fry, R. E. (1974). Toward an applied theory of experiential learning. M.I.T: Alfred P. Sloan School of Management.

Ministerio de Educación Nacional de la República de Colombia (2004). Estándares básicos de competencias ciudadanas. Formar para la ciudadanía ¡Sí es posible! Lo que necesitamos saber y saber hacer. Serie: Guías No. 6 . Bogotá: Mineducación.

Peña-López, I. (2016). ¿Eslabón o nodo? Cuatro redes de aprendizaje para el docente y el estudiante. Conferencia para la Inducción de profesores 2016 en Uniminuto, 12-14 de enero de 2016. Bogotá: Uniminuto.

Presidencia de la República de Colombia. Decreto 1038 de 2015: Por el cual se reglamenta la Cátedra de la Paz.

Servicio Nacional de Aprendizaje SENA. (2013). Proyecto Educativo Institucional PEI. Bogotá: SENA.
Approach to Program RAP. Geneva: Education \& Development department of the World Organization of the Scout Movement WOSM.

Rectoría UVD. (2016). Nueva maestría en Uniminuto. Recuperado de: http://www. uniminuto.edu/web/uvd/-/nueva-maestriaen-Uniminuto

Unipamplona. (2016). Recuperado de: http:// www.unipamplona.edu.co/unipamplona/ portalIG/home_83/publicacion/publicado/ index.htm

Uexternado. (2016). Recuperado de: http://www. uexternado.edu.co/esp/noticias/acuerdo_ sorbonne.html

Uniminuto. (2017). Recuperado de: http://www. Uniminuto.edu/web/fcc/modelo-ducativoy-metodología

The World Scout Bureau (2005). The Renewed

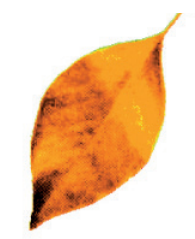

